Stefan Bösel · Karin Suttheimer

Freie Mitarbeit in den Medien 
Stefan Bösel · Karin Suttheimer

\section{Freie Mitarbeit \\ in den Medien}

Was Freelancer

wissen müssen

Westdeutscher Verlag 
Die Deutsche Bibliothek - CIP-Einheitsaufnahme

Ein Titeldatensatz für diese Publikation ist bei

Der Deutschen Bibliothek erhältlich

1. Auflage August 2002

Alle Rechte vorbehalten

(C) Westdeutscher Verlag GmbH, Wiesbaden 2002

Der Westdeutsche Verlag ist ein Unternehmen der Fachverlagsgruppe BertelsmannSpringer.

www.westdeutschervlg.de

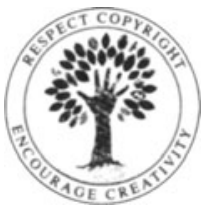

Das Werk einschließlich aller seiner Teile ist urheberrechtlich geschützt. Jede Verwertung außerhalb der engen Grenzen des Urheberrechtsgesetzes ist ohne Zustimmung des Verlags unzulässig und strafbar. Das gilt insbesondere für Vervielfältigungen, Übersetzungen, Mikroverfilmungen und die Einspeicherung und Verarbeitung in elektronischen Systemen.

Die in diesem Buch enthaltenen Hinweise und Empfehlungen sind mit keiner Verpflichtung oder Garantie irgendeiner Art verbunden. Autoren und Verlag übernehmen infolge dessen keine Verantwortung und werden keine daraus folgende oder sonstige Haftung übernehmen, die auf irgendeine Art aus der Benutzung des Materials oder Teilen daraus entsteht.

Die Wiedergabe von Gebrauchsnamen, Handelsnamen, Warenbezeichnungen usw. in diesem Werk berechtigt auch ohne besondere Kennzeichnung nicht zu der Annahme, dass solche Namen im Sinne der Warenzeichen- und Markenschutz-Gesetzgebung als frei zu betrachten wären und daher von jedermann benutzt werden dürften.

Umschlaggestaltung: Angela Kaleve-Dezius, Koblenz

Gedruckt auf säurefreiem und chlorfrei gebleichtem Papier

ISBN 978-3-531-13788-9 ISBN 978-3-322-90359-4 (eBook)

DOI 10.1007/978-3-322-90359-4 


\section{Danksagung}

\section{Vielen herzlichen Dank an:}

Barbara Emig-Roller, unsere Lektorin

für das uns entgegengebrachte Vertrauen

Veronika Mirschel, Referentin für Freie Mitarbeit, ver.di für ihr besonderes und selbstloses Engagement, ihre Zeit und Ideen

\section{Danke auch an:}

Gunther Haake, Projektleiter mediafon; Johannes Herz, Dipl.Finanzwirt; Dr. Sybille Schmid, Professorin an der HdM Stuttgart; Angela Brauner, Fachbereichssekretariat HdM Stuttgart; Bernd Engelhardt und Dr. Sabine Lißner, IHK Region Stuttgart; Susanne Dorer, ver.di; Goetz Buchholz, Autor; Herr Dr. Johannes Dürr; Sabine Lauster, Agentur Volles Programm; Dietmar Bosch, All Score Media; Dr. Jürgen Riethmüller; Martin Bethke

\section{Ganz besonderen Dank an:}

Erich und Ilse Suttheimer, Bernd Schmidt, Ingeborg und Matthias Bösel, Tina und Felix Güttner 


\section{Inhaltsverzeichnis}

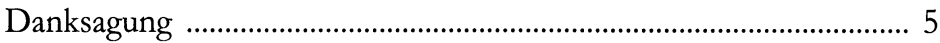

Inhaltsverzeichnis ….................................................................... 7

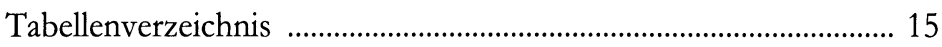

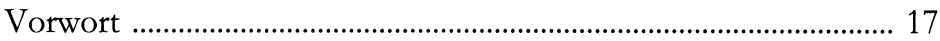

1. Was ist „freie Mitarbeit"? ........................................................ 23

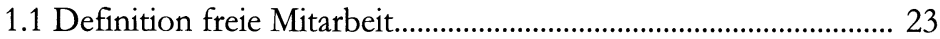

1.2 Vergleich: Festanstellung versus Selbstständigkeit....................... 24

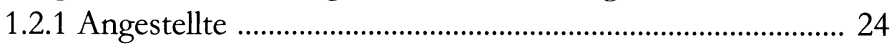

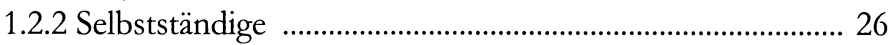

2. Allgemeine Überlegungen ..................................................... 29

2.1 Möglichkeiten, Risiken und Perspektiven ..................................... 29

2.2 Marktchancen: Situation, Volumen, Trends .............................. 34

2.2.1 Marktvolumen: Die aktuelle Arbeitsmarktsituation .......... 35

2.2.2 Wachstum des Marktes .................................................... 36

2.2.3 Die Zukunft: Allgemeine Trends am Arbeitsmarkt .......... 37

2.3 Haltung von Unternehmen gegenüber freier Mitarbeit ............. 38

2.3.1 Auftragsvergabe an Dritte: Outsourcing ........................... 38

2.3.2 Auswahlkriterien für freie Mitarbeiter ................................. 38

2.4 Erfolgskriterien freier Mitarbeit ............................................... 39

2.4.1 Die Motivation ................................................................. 39

2.4.2 Die fachliche Qualifikation ................................................ 40 
2.4.3 Die persönliche Qualifikation .............................................. 41

2.5 Eigene Zielsetzung …................................................................ 44

3. Rechtliche Grundlagen ................................................................. 45

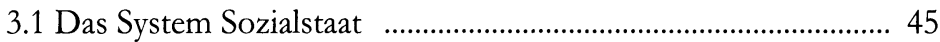

3.1.1 Die Sozialversicherungen und ihre Träger ......................... 46

3.1.1.1 Das System Krankenversicherung ........................... 47

3.1.1.2 Das System Pflegeversicherung ................................. 47

3.1.1.3 Das System Rentenversicherung ................................ 48

3.1.1.4 Das System Unfallversicherung ................................ 48

3.1.1.5 Das System Arbeitslosenversicherung ..................... 49

3.1.2 Gesetze gegen Missbrauch / Scheinselbstständigkeit ...... 49

3.2 Der eigene Status .................................................................... 50

3.3 Abgrenzungskriterien ................................................................ 52

3.4 Gesetzliche Definition: Selbstständige ........................................ 54

3.4.1 Selbstständige als Angehörige eines „Freien Berufes“ “.... 54

3.4.2 Selbstständige mit und ohne Gewerbeschein ................... 56

3.4.3 Arbeitnehmer ähnlich Selbstständige ................................. 58

3.4.4 Selbstständige Nebentätigkeit ............................................ 60

3.4.5 Selbstständige Nebentätigkeit von Studierenden .............. 60

3.4.6 Weitere Formen der Selbstständigkeit ................................. 61

3.5 Gesetzliche Definition: Arbeitnehmer .......................................... 61

3.5.1 Unbefristete/befristete Beschäftigte .................................... 64

3.5.2 Teilzeitbeschäftigte .............................................................. 64

3.5.3 Geringfügig Beschäftigte .................................................... 64

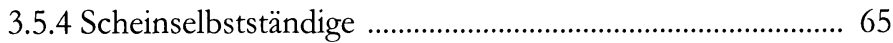

3.5.4.1 Gesetz zur Förderung der Selbstständigkeit ............ 66

3.5.4.2 Der Amtsermittlungsgrundsatz .................................. 66

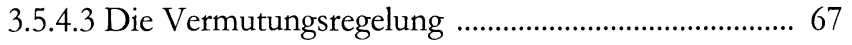

3.6 Mischverhältnisse: Selbstständig und Arbeitnehmer ...................6 68 
3.7 Das Anfrageverfahren ..................................................................... 68

3.7.1 Die telefonische Beratung ................................................. 69

3.7.2 Kriterien der Künstlersozialkasse ........................................ 69

4. Einstieg in die freie Mitarbeit ……………………………….... 71

4.1 Grundvoraussetzungen ............................................................... 71

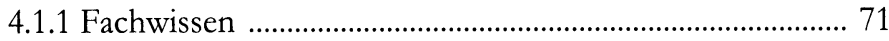

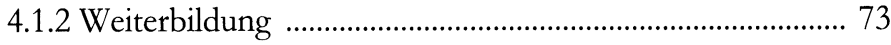

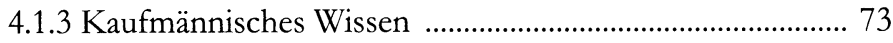

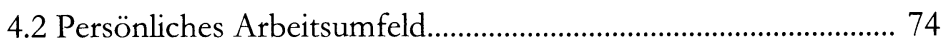

4.3 Berufsbezeichnung: Der passende Titel ...................................... 77

4.4 Professionelles Auftreten ........................................................... 78

4.4.1 Wirkung auf Auftraggeber ................................................... 78

4.4.2 Geschäftsausstattung ………………………………….... 78

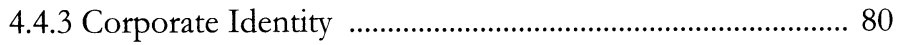

4.4.4 Referenzen als Erfolgsnachweis ...................................... 81

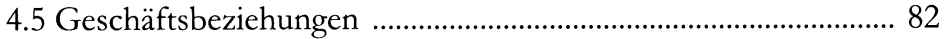

4.5.1 Bestehende Kontakte ......................................................... 82

4.5.2 Akquisition von Neukunden ............................................... 82

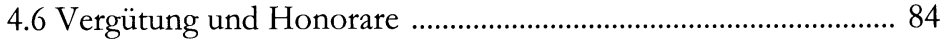

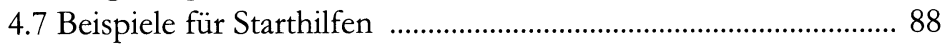

4.7.1 Das ERP-Eigenkapitalhilfedarlehen ...................................... 89

4.7.2 Das ERP-Existenzgründungsdarlehen................................... 90

4.7.3 Das DtA-Existenzgründungsdarlehen ............................... 91

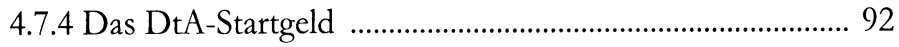

5. Auftragsabwicklung

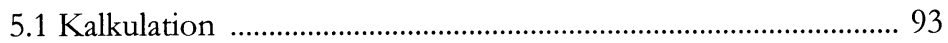

5.2 Angebotserstellung …………………………………………….... 94

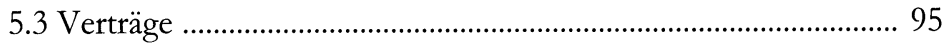


5.4 Rechte und Pflichten Selbstständiger ............................................. 97

5.5 Neutralität/Verschwiegenheitsverpflichtung ............................... 98

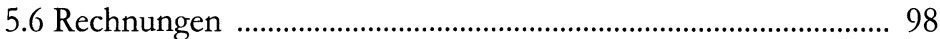

5.7 Das Mahnverfahren ............................................................... 99

5.7.1 Schritte vor dem Mahnverfahren ..................................... 100

5.7.2 Verzugszinsen ..................................................................... 101

5.7.3 Formale Voraussetzungen für ein Mahnverfahren ......... 101

5.7.4 Einleitung des Mahnverfahrens ...................................... 102

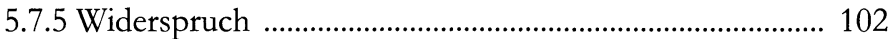

5.7.6 Der Vollstreckungsbescheid .............................................. 102

5.8 Controlling …................................................................................ 103

6. Versicherungen und Vorsorge ................................................ 107

6.1 Die Qual der Wahl ..................................................................... 107

6.2 Sozialversicherungen ................................................................ 109

6.2.1 Krankenversicherung (KV) .............................................. 109

6.2.1.1 Gesetzliche Krankenversicherung (GKV) ............. 110

6.2.1.1.1 Funktionsweise der GKV ............................... 111

6.2.1.1.2 Beitragsbemessung der GKV ......................... 111

6.2.1.1.3 Leistungsanspruch in der GKV ...................... 112

6.2.1.1.4 Familienversicherung in der GKV ................ 112

6.2.1.1.5 Beitragsbefreiung in der GKV ........................ 113

6.2.1.2 Private Krankenversicherung (PKV) ..................... 113

6.2.1.2.1 Funktionsweise der PKV ............................... 113

6.2.1.2.2 Beitragsbemessung der PKV .......................... 114

6.2.1.2.3 Leistungsanspruch in der PKV ...................... 115

6.2.1.2.4 Familienversicherung in der PKV .................. 115

6.2.1.2.5 Beitragsbefreiung in der PKV ........................ 116

6.2.1.2.6 Rücktrittsrecht ..................................................... 116

6.2.1.2.7 Rückkehr von der PKV zur GKV ................. 116

6.2.1.3 Krankentagegeld ........................................................ 117

6.2.1.4 Private Zusatzversicherung ..................................... 117 
6.2.2 Pflegeversicherung …….................................................... 117

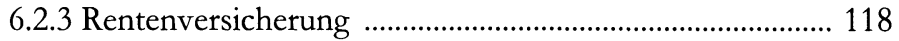

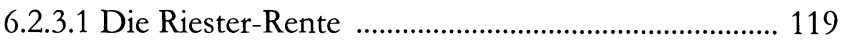

6.2.3.2 Beitragspflicht zur Rentenversicherung .................. 120

6.2.3.3 Freiwillige Rentenversicherung ............................... 120

6.2.3.4 Private Rentenversicherung ....................................... 121

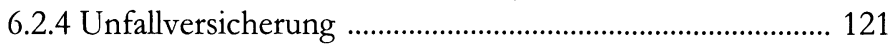

6.2.5 Arbeitslosenversicherung ............................................... 122

6.2.6 Die Künstlersozialkasse (KSK) ......................................... 122

6.2.6.1 Leistungen der KSK ................................................... 123

6.2.6.2 Aufnahmebedingungen der KSK ............................ 123

6.2.6.3 Antragverfahren der KSK ...................................... 124

6.3 Berufshaftpflichtversicherung ................................................. 124

6.4 Berufsrechtschutzversicherung ................................................ 125

6.5 Einkommensausfall-Versicherung .............................................. 126

7. Steuern und Finanzen ............................................................. 127

7.1 Rechte und Pflichten Angestellter ............................................ 127

7.2 Rechte und Pflichten Selbstständiger ........................................ 128

7.2.1 Die Anmeldung beim Finanzamt ..................................... 128

7.2.2 Steuervorauszahlungen .................................................... 129

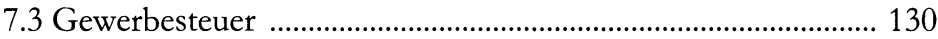

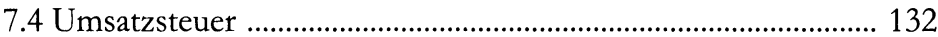

7.4.1 Umsatzsteuer bei internationaler Leistungserbringung .. 136

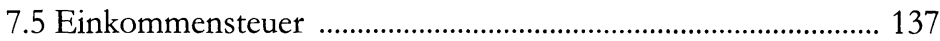

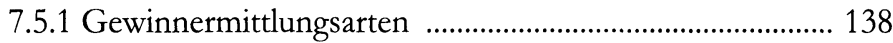

7.5.2 Betriebsvermögensvergleich ............................................... 138

7.5.3 Einnahme-/Überschussrechnung ...................................... 140

7.5.4 Das Wirtschaftsjahr .......................................................... 141

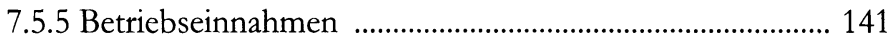

7.5.6 Betriebsvermögen ......................................................... 144 


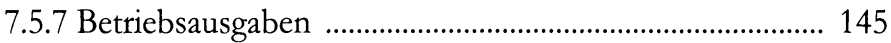

7.5.7.1 Abschreibungen ....................................................... 146

7.5.7.1.1 Die lineare Abschreibung ................................ 147

7.5.7.1.2 Die degressive Abschreibung ......................... 148

7.5.7.1.3 Vergleich lineare/degressive Abschreibung .. 148

7.5.7.2 Praktische Beispiele: Betriebsausgaben .................. 149

7.5.7.2.1 Pkw ............................................................ 149

7.5.7.2.2 Arbeitszimmer .................................................. 153

7.5.7.2.3 Telefonkosten ................................................. 155

7.5.7.2.4 Bewirtungsaufwendungen .............................. 155

7.5.7.2.5 Fachliteratur .................................................... 156

7.5.7.2.6 Kirchensteuer ................................................... 156

7.5.7.2.7 Abschreibung für Existenzgründer ................. 156

7.5.7.2.8 Sonderabschreibung ..................................... 157

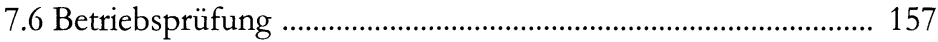

8. Fremde Hilfe und Beratung ………………………………..... 159

8.1 Staatliche Beratungsstellen ........................................................ 159

8.1.1 Bundesministerium für Wirtschaft und Technologie ..... 160

8.1.2 Arbeitsämter ................................................................... 160

8.1.3 Länderberatungsstellen .................................................... 160

8.2 Industrie und Handelskammer (IHK) ........................................ 161

8.3 Gewerkschaften - ver.di ........................................................... 162

8.3.1 Freienberatung mediafon .............................................. 164

8.3.2 Connexx.av ................................................................ 165

8.4 Unternehmensberatung ……………………………………… 166

8.5 Steuerberatung ……………………………………………….... 167

8.6 Finanzdienstleistung ………………………………………... 168

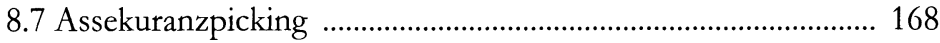

8.8 Rechtsberatung ……………………………………………..... 169

8.9 Sonstige …………………………………………………….... 169 
9.1 Was ist freie Mitarbeit - Checkliste zu Kapitel 1 171

9.2 Allgemeine Überlegungen - Checkliste zu Kapitel 2 172

9.3 Rechtliche Grundlagen - Checkliste zu Kapitel 3 172

9.4 Einstieg in die freie Mitarbeit - Checkliste zu Kapitel 4 172

9.5 Auftragsabwicklung - Checkliste zu Kapitel 5 172

9.6 Versicherungen und Vorsorge - Checkliste zu Kapitel 6 173

9.7 Steuern und Finanzen - Checkliste zu Kapitel 7 173

9.8 Fremde Hilfe und Beratung - Checkliste zu Kapitel 8 173

Anhang 175 


\section{Tabellenverzeichnis}

Tabelle 1: Erziehlbare Nettoeinkommen ........................................... 29

Tabelle 2: Vergleich feste und freie Mitarbeiter .............................. 35 im Raum Stuttgart

Tabelle 3: Wochenarbeitszeiten von Selbstständigen und ........... 43 Abhängigen

Tabelle 4: Beiträge zu den Sozialversicherungen ............................ 46

Tabelle 5: Die Sozialversicherungsträger …………………………. 47

Tabelle 6: IHK Beiträge von Kapitalgesellschaften ....................... 57

Tabelle 7: IHK Beiträge von Einzelunternehmen und ................. 57 Personengesellschaften

Tabelle 8: IHK Beiträge von Kleingewerbetreibenden ................. 58

Tabelle 9: Honorare und Stundensätze für freie Mitarbeiter ....... 85

Tabelle 10: Steuermesszahlen ............................................................ 131

Tabelle 11: Abschreibungsbetrag bei der linearen AfA ................ 148

Tabelle 12: Vergleich lineare und degressive Abschreibung .........149

Tabelle 13: Probleme beim Einstieg in die Selbstständigkeit ...... 164

Tabelle 14: Was belastet die Befragten in ihrer Arbeit? ............... 165

Tabelle 15: Zeitdauer der Selbstständigkeit der Befragten ............ 165

Tabelle 16: Einschätzung von mediafon ………………………..... 165 


\section{Vorwort}

Die projektbezogene „Freie Mitarbeit“ lässt sich aus der Medienbranche nicht mehr wegdenken. Filmproduktionen und Werbung, Computeranimationen, CD-ROM-, Event- und Tonproduktionen, Internetauftritte: in allen Bereichen der Medienindustrie kommen freie Mitarbeiter zum Einsatz. Viele Produktionsfirmen verfügen sogar über ein festes Kontingent an Personen, die kurzfristig bei Bedarf engagiert werden können. Der Vorteil für die Medienunternehmen: Schwankungen in der Auftragslage lassen sich durch freie Mitarbeiter kompensieren und sowohl der Umfang als auch die Art der angebotenen Leistungen ist nicht abhängig von der Anzahl oder Qualifikation der eigenen Angestellten - im Gegenteil, das Unternehmen bleibt in nahezu jeder Wirtschaftslage flexibel.

Der Einstieg in die projektbezogene freie Mitarbeit wirkt auf den ersten Blick im Vergleich zu einer Firmengründung relativ einfach: Ein Job wird angeboten, ausgeführt, eine Rechnung geschrieben und schon scheint ein neuer "freier Mitarbeiter" geboren. Doch - so einfach ist es nicht. Denn die freie Mitarbeit unterliegt, wie jede andere Beschäftigung auch, einer Reihe gesetzlicher Bestimmungen, Pflichten und natürlich auch Rechten. Sich als freier Mitarbeiter mit all diesen Themen zu beschäftigen, gestaltet sich - auch aus eigener Erfahrung gesprochen - mitunter sehr anstrengend. Kaum jemand bringt diese Zusatzqualifikation mit, sondern muss sich diese im Laufe der Zeit hart erarbeiten.

Dieser Umstand war für uns als Autoren der Hauptbeweggrund, dieses Buch zu schreiben. Da wir beide seit einiger Zeit Erfahrung mit dem Freelancen sammeln konnten, kam irgendwann der Gedanke, dieses Wissen anderen zu vermitteln und so 
einen gewissen Grunderfahrungsschatz zur Verfügung zu stellen, den sonst jeder angehende „Freie“ mühsam erarbeiten muss. Hinzu kommt, dass die meiste Fachliteratur sich mit dem Thema Existenzgründung im Sinne von Unternehmensgründung beschäftigt und so kaum in der Lage ist, auf die Spezifika der freien Mitarbeit - und schon gar nicht im noch spezielleren Medienbereich, in dem freie Mitarbeit einen sehr hohen Stellenwert besitzt - einzugehen.

Für wen ist dieses Buch? Eventuell wird auch der ein oder andere Medienentscheider oder Agenturgeschäftsführer dieses Werk lesen, um zu sehen, welche Vorteile sich für sein Unternehmen durch die Beauftragung von Freelancern ergeben - vielleicht auch, um einfach die Thematik besser zu verstehen. In erster Linie richtet es sich aber an Menschen, die sich damit beschäftigen, künftig ihre Brötchen als freie Mitarbeiter/innen zu verdienen - bereits in der Medienbranche tätige Angestellte genauso wie frisch gebackene Absolventen öffentlicher oder privater Bildungseinrichtungen und Quereinsteiger. Auch erfahrene Freelancer sollten einiges an Hintergrundwissen hinzu gewinnen. Im Laufe der Recherche haben auch wir unseren Erfahrungsschatz vergrößern können.

Ebenso sollten sich Menschen mit dem Ziel, irgendwann ein eigenes Medienunternehmen zu gründen, angesprochen fühlen. Denn für sie bietet die freie Mitarbeit eine zumindest temporäre Alternative, die sie ihrem Ziel ein gutes Stück näher bringen kann. Wer einmal erfolgreich freelanct, der ist auch von der Gründung des eigenen Unternehmens nicht allzu weit entfernt.

Was steht in diesen Buch und was nicht? Welche Erwartungen können Sie als Leser haben? Dieses Werk versucht eine möglichst breite Wissenspalette abzudecken, welche speziell für die professionelle freie Mitarbeit in der Medienbranche nötig ist. Es erhebt aber nicht den Anspruch, ,allwissend“ zu sein. So wird für Profis z.B. der Gang zum Steuerberater auch nach dem Lesen von Kapitel 7 „Steuern und Finanzen" nicht völlig überflüssig werden, aber mit Sicherheit dazu beitragen, beratende Spezialisten wie z.B. einen Steuerberater besser zu verstehen und auch besser zu nutzen. An etlichen Stel- 
len wird aber auf in der freien Mitarbeit und der Medienbranche relevanten Fälle soweit eingegangen, dass viele Probleme selbstständig gelöst werden können und fremde Beratung nicht immer in Anspruch genommen werden muss.

Wie ist das Buch aufgebaut? Wir haben das Buch so aufgebaut, wie wir glauben, dass sich der informationssuchende Neuling dem Thema „freie Mitarbeit in den Medien“ nähert. Wer sich zu den bereits Fortgeschrittenen zählt, kann seinen Einstieg nach Belieben auch in einem der hinteren Kapitel suchen. Dem entsprechend ist auch der Aufbau des Buches:

In Kapitel 1 „Was ist freie Mitarbeit“ werden grundsätzliche Begrifflichkeiten definiert und die Unterschiede zwischen selbstständiger und nichtselbstständiger Arbeit aufgezeigt.

Dann wird mit Kapitel 2 „Allgemeine Überlegungen“ Basiswissen vermittelt. Es erklärt, was die freie Mitarbeit als Alternative zur Festanstellung grundsätzlich bedeutet und welche Personen sich für eine freie Mitarbeit eignen. Definition und Vergleiche von freier Mitarbeit mit dem Angestelltenstatus helfen, zwischen diesen Arbeitsformen zu unterscheiden. Chancen, Risiken und Möglichkeiten freier Mitarbeit werden aufgezeigt, die Situation am Arbeitsmarkt, Verdienstmöglichkeiten erklärt und auch schon hier zu Beginn wird ganz speziell auf die Medienbranche eingegangen. Dieses Kapitel bietet die Grundlage für eine persönliche Entscheidung für bzw. gegen die freie Mitarbeit.

Beginnend mit Kapitel 3 „Rechtliche Grundlagen“ wird das in den Kapiteln 1 und 2 beschriebene Basiswissen nach und nach vertieft: In Kapitel 3 werden die gesetzlichen Bestimmungen, auf der die freie Mitarbeit basiert - zum einen in der abhängigen, zum anderen in der nicht abhängigen, also selbstständigen Beschäftigung erläutert. Dabei werden die rechtlichen Grundlagen nicht nur beschrieben, sondern es wird auch der Zusammenhang zum bestehenden Rechtssystem der Bundesrepublik Deutschland erklärt.

Nach Klärung des eigenen rechtlichen Status' kann mit der Aufnahme der Tätigkeit als freier Mitarbeiter begonnen werden. Ka- 
pitel 4 „Einstieg in die freie Mitarbeit“ zeigt sich dann praxisorientiert: Grundvoraussetzungen, wie z.B. das nötige Wissen und die Arbeitsmittel, Starthilfen, professionelles Auftreten und der Aufbau von Geschäftsbeziehungen bis hin zur möglichen Vergütung werden beschrieben.

Kapitel 5 „Auftragsabwicklung“ geht noch einen Schritt weiter in die Praxis und zeigt, wie freie Mitarbeiter ihre Aufträge abwickeln: Von der Projektkalkulation über die Angebotserstellung und den eventuell benötigten Verträgen bis hin zu den sich daraus ergebenden Rechten und Pflichten. Es wird erklärt, wie Rechnungen an Auftraggeber gestellt werden und wie die Zahlungseingänge mittels Mahnverfahren erwirkt werden können. Darüber hinaus werden im letzten Punkt „Controlling“ der Bereich Erfolgskontrolle und die dafür nötigen Maßnahmen näher gebracht.

Ist die freie Mitarbeit erst einmal erfolgreich, gilt es, sich auch entsprechend abzusichern: Kapitel 6 „Versicherungen und Vorsorge" vertiefen das Wissen in punkto Sozialversicherungen (speziell die verschiedenen Krankenversicherungsmodelle finden hier Berücksichtigung), Haftpflicht-, Rechtschutz- sowie Einkommensausfallversicherungen.

Kapitel 7 „Steuern und Finanzen“ geht zunächst auf die steuerrechtlichen Aspekte der selbstständigen freien Mitarbeit ein, zeigt dann, was dem Finanzamt gegenüber nachzuweisen ist, und wie verschiedene, für die freie Mitarbeit relevante, Ausgaben steuerlich geltend gemacht werden können.

In Kapitel 8 „Fremde Hilfe und Beratung“ werden zum einen Möglichkeiten aufgezeigt, derer sich freie Mitarbeiter bedienen können, um z.B. reibungsloser und professioneller arbeiten zu können. Zum anderen beschreibt Kapitel 8 aber auch Anlaufstellen für freie Mitarbeiter, wenn die Arbeit einmal nicht reibungslos läuft.

Abschließend zieht Kapitel 9 noch einmal ein kurzes Resümee und bietet mittels einer Checkliste die Möglichkeit, alle für die freie Mitarbeit notwendigen Schritte Revue passieren zu lassen und auf diese Weise zu verifizieren. 
Im Anhang findet sich ein nützliches Sammelsurium von Adressen, Internetlinks, Musterbeispielen und vieles mehr.

Noch ein Wort zum Abschluß: wie immer gestaltet sich auch in diesem Buch die Suche nach der korrekten Personen-Ansprechform als schwierig. Freie Mitarbeiter oder Freie Mitarbeiterin? Freie/r Mitarbeiter/in? Freie MitarbeitInnen? Wir haben uns auch mit dieser Frage auseinandergesetzt und uns schliesslich dafür entschieden, die für den Lesefluss geeigneten Titel zu wählen. Für das gesamte Werk gilt aber: unabhängig vom Titel sind immer, ebenso Frauen wie auch Männer angesprochen.

Viel Spaß beim Lesen!

Karin Suttheimer und Stefan Bösel im Mai 2002

P.S.: Kritik und Anregungen entweder an den Verlag oder direkt an:

freelance@suttheimer.com

(Karin Suttheimer)

freelance@webdeluxe.de

(Stefan Bösel) 